\title{
Nutrição e doenças cardiovasculares: os marcadores de risco em adultos
}

\author{
Nutrition and cardiovascular diseases: the \\ risk markers in adults
}

Luiza Carla Vidigal CASTRO'

Sylvia do Carmo Castro FRANCESCHINI ${ }^{2}$

Sílvia Eloíza PRIORE²

Maria do Carmo Gouveia PELÚZIO²

\section{R E S U M O}

As doenças cardiovasculares contribuem significativamente, como grupo causal, para a taxa de mortalidade em todas as regiões brasileiras, principalmente na Região Sudeste. Além disso, constituem uma das principais causas de permanência hospitalar prolongada e são responsáveis pela principal alocação de recursos públicos em hospitalizações no Brasil. O ônus econômico das doenças cardiovasculares tem crescido exponencialmente nas últimas décadas. O risco de se desenvolver doença cardiovascular é avaliado com base na análise conjunta de características que aumentam a chance do indivíduo vir a apresentar a doença. O conhecimento desses fatores associados ao risco é de grande importância para o estabelecimento de estratégias de prevenção. Este artigo em questão revisa os principais marcadores de risco para doenças cardiovasculares em adultos, relacionados à nutrição, como os antropométricos, dietéticos e bioquímicos. Além disso, enfatiza o impacto destas morbidades na sociedade, bem como a necessidade de serem estabelecidas medidas de prevenção primária no controle das mesmas.

Termos de indexação: doenças cardiovasculares, nutrição, marcadores de risco, morbidade, adulto.

\section{A B S T R A C T}

The cardiovascular diseases contribute significantly as a death cause in all Brazilian regions, mainly the Southeast. Besides, they constitute one of the main causes of permanence in hospital and are responsible for the main

\footnotetext{
1 Programa de Pós-Graduação em Ciência da Nutrição, Departamento de Nutrição e Saúde, Universidade Federal de Viçosa. Campus Universitário, s/n, 36571-000, Viçosa, MG, Brasil. Correspondência para/Correspondece to: L.C.V. CASTRO. E-mail: luizavidigal@ hotmail.com

2 Departamento de Nutrição e Saúde, Universidade Federal de Viçosa, Viçosa, MG, Brasil.
} 
allocation of public resources in hospitalizations in Brazil. Consequently, the economic cost of cardiovascular diseases has increased in the last decades. The risk of someone developing cardiovascular disease is evaluated through analysis of the characteristics increasing that individual's chance to develop the disease. The knowledge of those factors associated to the risk is important in order to establish prevention strategies. This article revises the main risk markers for cardiovascular diseases in adults related to the nutrition, as the anthropometrics, dietary and biochemical. Besides, it emphasizes the impact of these diseases in society, as well as the need to take importance of establishing measures of primary prevention to control them.

Index terms: cardiovascular diseases, nutrition, risk markers, morbidity, adult.

\section{N T R O D U Ç Ã O}

As mudanças que vêm ocorrendo nas sociedades dos países em desenvolvimento, dentre eles o Brasil, acompanham-se de modificações importantes no perfil de morbidade e de mortalidade. As doenças não transmissíveis representam, atualmente, importante problema de saúde pública nesses países. O Brasil, além de enfrentar o problema ainda não resolvido das doenças infecciosas e parasitárias, defronta-se com as doenças crônicas, de alto custo social e mais difícil prevenção ${ }^{1}$.

As doenças cardiovasculares contribuem significativamente como grupo causal de mortalidade em todas as regiões brasileiras. De acordo com o Ministério da Saúde ${ }^{2}$ a Região Sudeste possui o maior coeficiente de mortalidade por doenças do aparelho circulatório (207 mortes por 100 mil habitantes), enquanto a média brasileira é de 169 mortes/100 mil habitantes.

O ônus econômico das doenças cardiovasculares cresceu exponencialmente nas últimas décadas. Em 2000, as doenças cardiovasculares foram responsáveis pela principal alocação de recursos públicos em hospitalizações no Brasil e foram a terceira causa de permanência hospitalar prolongada. Entre 1991 e 2000, os custos hospitalares atribuídos às doenças cardiovasculares aumentaram cerca de $176 \%{ }^{2,3}$.

O risco de se desenvolver doenças crônico-degenerativas é avaliado com base na análise conjunta de características que aumentam a probabilidade de um indivíduo vir a apresentar a doença. É necessário distinguir o conceito de fator de risco (agente causal) de marcador de risco (associação com maior risco, porém sem causalidade estabelecida $)^{4,5}$. O conhecimento tanto dos fatores quanto dos marcadores de risco é fundamental para o estabelecimento de estratégias de prevenção das doenças cardiovasculares $^{4,6}$.

Entre os fatores de risco de maior probabilidade para o desenvolvimento das doenças cardiovasculares (DCV) estabelecidos desde o estudo de Framinghan, destacam-se o fumo, a hipertensão arterial, as dislipidemias e o diabetes mellitus $^{4}$. A obesidade e a inatividade física foram positivamente associados com o risco de desenvolver DCV, constituindo-se nos fatores de risco mais significativos ${ }^{4}$. Da mesma forma, o National Cholesterol Education Program (NCEP), a American Heart Association (AHA), a Sociedade Européia de Cardiologia e a Sociedade Brasileira de Cardiologia têm assinalado a fundamental implicação da obesidade, da dieta e da inatividade física no risco cardiovascular ${ }^{3,7,8}$.

Este trabalho tem como objetivo revisar os principais marcadores de risco para doenças cardiovasculares em adultos, relacionados à nutrição, como os antropométricos, dietéticos e bioquímicos.

\section{Marcadores antropométricos}

A Organização Mundial de Saúde indica o uso da antropometria para a vigilância dos fatores de risco das doenças crônicas. Além do peso e da altura, recomenda a medida da cintura e do quadril como forma de avaliar a deposição da 
gordura abdominal ${ }^{9}$. Esses parâmetros antropométricos têm a vantagem de apresentar fácil mensuração e obtenção a baixo custo, podendo ser utilizados tanto na saúde pública quanto na clínica.

O Índice de Massa Corporal (IMC) $\left(\mathrm{kg} / \mathrm{m}^{2}\right)$ - acima de 25 , que caracteriza o sobrepeso, está associado a maior risco de desenvolvimento de morbidades crônicas não transmissíveis, sendo este gradativo e contínuo ${ }^{10}$. Entretanto, como os indivíduos diferem em relação à composição corporal e localização da gordura, o uso do IMC deve ser associado a medidas da distribuição de gordura, como forma de melhor predizer o risco ${ }^{10,11}$. Os homens tendem a ter maior proporção de gordura abdominal, conferindo-lhes o chamado padrão masculino ou andróide de distribuição de gordura. Por outro lado, as mulheres tendem a ter maior quantidade de gordura na região glútea, apresentando o padrão feminino ou ginóide de distribuição de gordura corporal ${ }^{9}$. Este padrão pode ser avaliado pela razão entre a circunferência da cintura e circunferência do quadril, conhecido como razão cintura/quadril $(R C Q)^{12}$, bem como pela razão cintura/altura $(R C A)^{13}$ e circunferência da cintura ${ }^{14}$. A RCQ e a circunferência da cintura (CC), são as medidas mais utilizadas para estimar a gordura abdominal que, por sua vez, relaciona-se à quantidade de tecido adiposo visceral ${ }^{15}$.

Estudo epidemiológico ${ }^{16}$ mostrou que a obesidade central estava associada com a hipertensão arterial, importante fator de risco das doenças cardiovasculares. Da mesma forma, o excesso de gordura na região abdominal (adiposidade central) pode ter maior capacidade preditiva que a massa corporal total para o infarto do miocárdio e o acidente vascular cerebral ${ }^{17}$.

Os pontos de corte de RCQ - como preditor de doenças crônicas - mais utilizados para homens $(>1,0 ;>0,95)$ e mulheres $(>0,80 ;>0,80)$, baseiam-se em estudos epidemiológicos suecos e canadenses, respectivamente ${ }^{18}$.

No Brasil, estudo desenvolvido por Pereira et al. ${ }^{19}$ definiu os melhores pontos de corte para a
RCQ, usando-os como preditores da hipertensão arterial. Os pesquisadores estudaram uma amostra de 3282 indivíduos, sendo $43,1 \%$ do sexo masculino e $56,9 \%$ do sexo feminino. Os melhores pontos de corte encontrados foram 0,80 para mulheres e 0,95 para homens. Além disso, os pesquisadores verificaram que a RCQ apresentou menor correlação com o IMC, quando comparada com a razão cintura/altura e circunferência da cintura. Entretanto, a RCQ apresentou melhor capacidade preditiva de hipertensão arterial, o que evidencia a importância de sua utilização na discriminação de indivíduos em risco de doenças crônicas. Já Lerario et al. ${ }^{20}$, estudando a comunidade nipo-brasileira na faixa etária de 40 e 79 anos, encontraram uma razoável correlação entre a RCQ e o IMC nos homens $(r=0,48$; $p<0,000)$ e nas mulheres $(r=0,52 ; p<0,000)$.

A RCQ elevada ( $>1,0$ nos homens e $>0,85$ nas mulheres) tem sido utilizada como medida clínica para avaliar indivíduos com acúmulo de gordura abdominal ${ }^{9,10}$. Porém, a utilização da circunferência da cintura, apenas, tem mostrado uma boa correlação com a gordura abdominal associada com o processo saúde-doença. Alterações da CC refletem alterações nos fatores de risco para doenças cardiovasculares e outras formas de doenças crônicas ${ }^{10}$.

Em estudo realizado entre mulheres negras americanas, Conway et al. ${ }^{21}$ verificaram que a circunferência da cintura foi a medida antropométrica que melhor se correlacionou com a distribuição visceral de gordura. Segundo Lean et al. ${ }^{14}$ e Han et al..$^{22}$, os pontos de corte para a CC devem ser $94 \mathrm{~cm}$ (homens) e $80 \mathrm{~cm}$ (mulheres). De acordo com a Organização Mundial da Saúde ${ }^{10}$, os riscos de complicações metabólicas são aumentados quando a CC é superior a $94 \mathrm{~cm}$ (homens) e $80 \mathrm{~cm}$ (mulheres) e muito aumentados quando maior que $102 \mathrm{~cm}$ (homens) e $88 \mathrm{~cm}$ (mulheres).

Estudo ${ }^{11}$ envolvendo 341 homens e mulheres brancos, com idade entre 18 e 88 anos e IMC entre 15,9 e $47,7 \mathrm{~kg} / \mathrm{m}^{2}$, submetidos à imagem por ressonância magnética, verificou que, 
independente da idade e do sexo, a combinação dos indicadores antropométricos IMC e CC explicam maiores variações da gordura visceral, abdominal subcutânea e não abdominal quando comparados com o uso de um dos indicadores isoladamente. O IMC foi mais fortemente correlacionado com a gordura não abdominal e abdominal subcutânea. Em relação à gordura visceral, observou-se uma melhor correlação com a CC. Para cada categoria de IMC estudada, um aumento da circunferência da cintura foi associado com um aumento da gordura visceral. Enfatiza-se, assim, a importância do uso concomitante destes indicadores na prática clínica.

No Brasil, estudo recente ${ }^{23}$ avaliou a eficácia em usar-se a circunferência da cintura para identificar sobrepeso $\left(\mathrm{IMC}>25 \mathrm{~kg} / \mathrm{m}^{2}\right)$, obesidade $\left(\mathrm{IMC}>30 \mathrm{~kg} / \mathrm{m}^{2}\right)$ e hipertensão arterial em mulheres com idade entre 15 e 59 anos. Tanto a CC quanto o IMC e a RCQ mostraram correlação positiva com hipertensão arterial, sendo que a correlação foi similar para a CC e IMC e mais fraca para a RCQ. As CC $>0,80 \mathrm{~cm}$ e $>0,88 \mathrm{~cm}$, respectivamente, discriminaram com exatidão $89,8 \%$ de mulheres com sobrepeso e $88,5 \%$ com obesidade. A obesidade abdominal (CC $>0,88 \mathrm{~cm})$ esteve associada significativamente com a hipertensão na análise multivariada $(\mathrm{OR}=2,88$, IC $95 \%: 1,77-4,67)^{23}$.

\section{Marcadores da dieta}

Diversos estudos têm evidenciado a relação entre características qualitativas e quantitativas da dieta e ocorrência de enfermidades crônicas, entre elas, as doenças cardiovasculares ${ }^{24,25,26}$. Os hábitos alimentares apresentam-se como marcadores de risco para doenças cardiovasculares, na medida em que o consumo elevado de colesterol, lipídios e ácidos graxos saturados somados ao baixo consumo de fibras, participam na etiologia das dislipidemias, obesidade, diabetes e hiper-tensão24,27,28,29,30.

Os componentes nutricionais com maior influência no perfil lipídico de indivíduos saudáveis são: a ingestão de gordura total, a composição de ácidos graxos da dieta, o colesterol, a fonte de proteínas animal/vegetal, fibras e compostos fitoquímicos $25,26,31$. Entretanto, uma vez que a alimentação diária é complexa, contendo diversos alimentos e, conseqüentemente, nutrientes, ainda não foi possível elucidar ou quantificar precisamente o impacto específico da alimentação no risco do desenvolvimento de doenças cardiovasculares ${ }^{25}$.

Diversos estudos têm demonstrado que modificações na composição lipídica da dieta podem promover alterações nos níveis séricos de colesterol, evidenciando o efeito da dieta nos níveis de colesterol plasmático, que pode ser significativamente modificado pela quantidade e qualidade dos ácidos graxos ingeridos $29,32,33,34$. Os ataques cardíacos e a aterosclerose são raros em populações que apresentam baixos níveis de colesterol plasmático. Porém, quando essas adotam dieta tipicamente "ocidental", apresentam, como conseqüência, níveis plasmáticos de colesterol elevados, aumentando a incidência de doenças isquêmicas cardíacas ${ }^{35}$.

Parada et al. ${ }^{28}$, ao estudarem a relação entre hábitos alimentares e níveis de colesterol sérico em uma população suburbana da Argentina, verificaram que, ao longo de treze anos, houve uma redução no consumo de carnes vermelhas (bovina, suína e ovina), ovos, leite e derivados integrais como a manteiga. Concomitantemente, observaram um aumento no consumo de aves, peixes, leite e seus derivados desnatados e azeites (principalmente de semente de girassol). A análise do colesterol total plasmático, com doze horas de jejum, mostrou que houve uma redução dos níveis séricos de colesterol, coincidente com as modificações dietéticas.

Fornes et al. ${ }^{25}$, estudando a relação entre a freqüência de consumo de alimentos e os níveis séricos de lipoproteínas em população do município de Cotia, SP, observaram que o controle dietético pode reduzir os riscos de doenças cardiovasculares. Neste estudo, o consumo de carnes processadas, aves, carnes vermelhas, ovos 
e leite/derivados correlacionou-se positiva e significantemente com as frações LDL-colesterol (aumento de $16,6 \mathrm{mg} / \mathrm{dL}, 14,5 \mathrm{mg} / \mathrm{dL}, 11,1 \mathrm{mg} / \mathrm{dL}$, $5,8 \mathrm{mg} / \mathrm{dL}$ e $4,6 \mathrm{mg} / \mathrm{dL}$, respectivamente) enquanto o consumo de frutas e hortaliças mostrou correlação inversa (redução de 5,2 e 5,5mg/dL, respectivamente).

A análise por escores da freqüência de consumo de alimentos pode ser um método de escolha para avaliar a qualidade da dieta e seu efeito potencial nos níveis séricos de colesterol total e lipoproteínas de baixa densidade ${ }^{36}$. Em estudo ${ }^{36}$ realizado na área metropolitana de São Paulo, os autores classificaram os alimentos, registrados por meio de inquérito de consumo alimentar, em sete categorias de freqüência de consumo e em dois grupos. Estes, de acordo com o potencial de risco para doenças cardiovasculares, eram classificados como: alimentos de risco (grupo 1 - produtos lácteos integrais, gorduras de origem animal, margarinas - devido ao conteúdo em ácidos graxos trans -, alimentos fritos, carnes e produtos derivados, ovos) e alimentos protetores ou não considerados de risco (grupo 2 - frutas e sucos naturais, hortaliças, leguminosas, cereais e derivados). Os autores encontraram correlação positiva entre ingestão de alimentos considerados de risco para doenças cardiovasculares e os níveis séricos de colesterol total e fração LDL-colesterol; a correlação foi negativa entre estes níveis e a ingestão de alimentos protetores ou não considerados de risco.

O Estudo dos Sete Países ${ }^{37}$, envolvendo homens com idade entre 40 e 59 anos, acompanhados durante 15 anos, foi o primeiro a demonstrar que o consumo de ácidos graxos saturados apresentava forte correlação com os níveis plasmáticos de colesterol. Países com maior consumo de gordura saturada (superior a 15\% da energia diária) apresentaram maiores concentrações de colesterol e maior mortalidade por doença arterial coronária. A partir destes estudos iniciais, foram identificados outros componentes alimentares e alimentos específicos que têm uma ligação positiva ou negativa com o risco de doenças cardiovasculares.
Embora as evidências epidemiológicas demonstrem que um baixo consumo de gordura está associado a níveis mais baixos de colesterol e menor incidência de cardiopatias coronarianas, parece que o tipo de gordura presente numa dieta moderada neste nutriente (25\% a 30\% da energia total ingerida diariamente), é mais importante que a quantidade de gordura ingerida. Substituindo-se a gordura saturada por insaturada, verifica-se que os níveis séricos de lipídios e colesterol são substancial e consistentemente reduzidos na maioria dos $\operatorname{casos}^{26,33,34}$.

Entretanto, a remoção da gordura saturada da dieta é duas vezes mais eficaz que o acréscimo da mesma quantidade de lipídios insaturados para reduzir o colesterol do sangue ${ }^{38}$. Santo ${ }^{39}$ enfatiza que "a ingestão de ácidos graxos saturados está fortemente correlacionada com o nível de colesterol, e este com a incidência de infarto do miocárdio fatal e não fatal". Este autor assinala que em países com alto índice de doença coronariana, predomina a alimentação com alto teor de gordura saturada. Por outro lado, em países com baixa incidência de doença coronariana, os hábitos alimentares incluem principalmente óleos vegetais e baixo consumo de produtos lácteos.

Nem todos os ácidos graxos saturados afetam as concentrações de colesterol da mesma maneira. Os ácidos graxos saturados, com exceção do esteárico, aumentam os níveis séricos de todas as lipoproteínas, principalmente as de baixa densidade (colesterol-LDL), uma vez que reduzem a síntese e atividade dos receptores $L D L$, pela diminuição da expressão de RNA mensageiro e da fluidez da membrana26,40. Dessa forma, os ácidos graxos saturados aumentam os níveis de LDL-colesterol por meio da redução de sua depuração da circulação. A elevação da fração LDL do colesterol sangüíneo irá favorecer o depósito lipídico nas paredes dos vasos, ocasionando o aparecimento de placas ateromatosas. Como conseqüência, aumentam as probabilidades de um ataque cardíaco.

Em um experimento realizado com humanos, a quantidade de receptores para LDL 
aumentou 10,5\% após ingestão de dieta pobre em gordura saturada. Associou-se a esse aumento redução correspondente de $11,8 \%$ nos níveis séricos de LDL-colesterol. Portanto, parece que a redução de gordura saturada da dieta está relacionada com aumento na expressão de receptores para LDL de amplitude similar à redução de LDL-colesterol ${ }^{40}$. A gordura polinsaturada e monoinsaturada estão relacionadas a menores riscos de enfermidades cardíacas. O ácido graxo esteárico, apesar de ser saturado, não é considerado aterogênico, uma vez que, dentro do organismo, é rapidamente convertido a ácido oléico (monoinsaturado) 26,40,41.

Mattson et al. ${ }^{42}$ verificaram uma relação linear entre o colesterol da dieta e sangüíneo; observaram que cada $100 \mathrm{mg}$ de colesterol/ $1000 \mathrm{kcal}$ consumidas resultou em um aumento no colesterol plasmático de $12 \mathrm{mg} / 100 \mathrm{~mL}$. O colesterol da dieta contribui com aproximadamente $15 \%$ na síntese do colesterol endógeno ${ }^{43}$. Entretanto, a redução de $100 \mathrm{mg}$ no colesterol da dieta resulta numa queda de apenas $5 \mathrm{mg}$ no colesterol sérico, quando a gordura da dieta não é alterada; ou seja, o colesterol ingerido não tem tanta influência no colesterol sérico, como tem o consumo de gordura saturada, mono ou polinsaturada ${ }^{38}$. Por outro lado, a redução da colesterolemia, mesmo que pequena, parece ser eficiente na diminuição dos índices de mortalidade por doenças cardiovasculares ${ }^{38}$.

\section{Marcadores bioquímicos}

Estudos epidemiológicos longitudinais têm demonstrado que existe uma correlação direta entre os níveis de colesterol plasmático e triglicerídeos e o aumento de doenças cardiovasculares $^{44,45}$. Essa correlação depende, particularmente, da concentração das lipoproteínas (notadamente LDL e HDL) que transportam o colesterol na corrente sangüínea ${ }^{46}$.

Os mecanismos pelos quais as diversas lipoproteínas se relacionam com as doenças cardiovasculares são complexos, envolvendo a formação de células espumosas, resposta inflamatória, alterações plaquetárias, alterações do endotélio e formação de placas ateroscleróticas $^{3,46}$. Entre os fatores de risco envolvidos neste processo tem-se as dislipidemias, o diabetes, a hipertensão arterial, o tabagismo e concentrações elevadas de homocisteína ${ }^{26}$.

As dislipidemias podem ser classificadas, do ponto de vista laboratorial, em hipercolesterolemia isolada (aumento do colesterol total e/ou da fração LDL-colesterol), hipertrigliceridemia isolada (aumento dos triacilgliceróis), hiperlipidemia mista (aumento do colesterol total e dos triacilgliceróis) e diminuição isolada do HDL-colesterol ou associada ao aumento dos triacilgliceróis ou LDL-colesterol ${ }^{3}$. Os valores de referência para o diagnóstico de dislipidemias em adultos são: colesterol total $\geq 240 \mathrm{mg} / \mathrm{dL}$ (alto), 200-239mg/dL (limítrofe); LDL-colesterol $\geq 190 \mathrm{mg} / \mathrm{dL}$ (muito alto); 160-189mg/dL (alto); 130-159mg/dL (limítrofe); HDL-colesterol <40mg/dL (baixo); triacilgliceróis $\geq 500 \mathrm{mg} / \mathrm{dL}$ (muito alto), $200-499 \mathrm{mg} / \mathrm{dL}$ (alto) e 150-200mg/dL (limítrofe) ${ }^{8}$.

A doença cardíaca coronariana é rara em sociedades com concentrações plasmáticas de colesterol total abaixo de $180 \mathrm{mg} / \mathrm{dL}^{26}$. Uma meta-análise ${ }^{47}$ realizada recentemente relatou que, para cada redução de $10 \%$ de colesterol plasmático, o risco de mortalidade por doença cardiovascular pode ser reduzido em $15 \%$ e o risco total de mortalidade em $11 \%$.

Além das dislipidemias, há indícios de que níveis elevados de lipoproteína (a), homocisteína e proteína $C$ reativa estão associados com maior risco de doenças cardiovasculares, o que permite classificá-los como possíveis marcadores de risco. A lipoproteína (a) é rica em colesterol e semelhante à lipoproteína LDL e atua na inibição da fibrinólise e da síntese de plasmina, o que lhe confere uma propriedade pró-aterogênica. A homocisteína é um aminoácido derivado do metabolismo da metionina e sua elevação tem sido associada à disfunção do endotélio, trombose e maior gravidade da aterosclerose $e^{3,48}$. Entretanto, ainda não há consenso quanto a eficácia em reduzir-se 
a concentração dessas substâncias para reduzir-se o risco de desenvolvimento das doenças citadas; portanto, mais estudos são necessários para elucidar o papel dessas substâncias no processo aterosclerótico.

A proteína $C$ reativa é um marcador da inflamação; sua concentração sangüínea acima de $1,9 \mathrm{mg} / \mathrm{dL}$, também tem sido associada a um alto risco de doença cardiovascular, uma vez que um processo inflamatório crônico está envolvido na aterosclerose ${ }^{49,50}$.

Como os indivíduos podem apresentar múltiplos fatores de risco, é possível predizer o risco absoluto de se desenvolver doença cardíaca coronariana (angina, infarto agudo do miocárdio, morte) utilizando-se os escores de risco de Framingham. Neste método, de acordo com o sexo, são atribuídos pontos para idade, colesterol total e fração HDL, fumo e pressão sangüínea. A fração LDL-colesterol é considerada fator causal e independente da aterosclerose. O risco absoluto é estratificado em baixo risco (risco absoluto de eventos $<10 \%$ em 10 anos), médio risco (risco absoluto de eventos entre $10 \%$ e $20 \%$ em 10 anos) e alto risco (risco absoluto de eventos $\geq 20 \%$ em 10 anos). O diabetes é considerado risco equivalente à aterosclerose. Geralmente, a classificação de baixo risco inclui indivíduos com um fator de risco (exceto diabetes) além do colesterol $\mathrm{LDL}>160 \mathrm{mg} / \mathrm{dL}$; a de médio risco inclui indivíduos com dois fatores de risco; e a de alto risco, indivíduos com mais de dois fatores de risco $3,8,26$.

Entre as estratégias de prevenção primária das doenças cardiovasculares destacam-se as mudanças no estilo de vida, entre elas, a redução na ingestão de gordura saturada, controle do peso corporal e prática de atividade física. Estas mudanças são enfatizadas em todos os níveis de risco (baixo, médio e alto risco) ${ }^{38}$.

\section{O N CLUS Ã O}

De acordo com o exposto no presente artigo, conclui-se que a avaliação do estado nutricional é de grande utilidade e importância para o estabelecimento de estratégias de intervenção visando à prevenção de doenças cardiovasculares, uma vez que os marcadores de risco relacionados à nutrição, como os antropométricos, dietéticos e bioquímicos, podem ser modificados com a adoção de estilo de vida saudável e controle do peso corporal.

\section{R E F E R Ê N C I A S}

1. Barata RCB. O desafio das doenças emergentes e a revalorização da epidemiologia descritiva. Rev Saúde Publica 1997; 31(5):531-7.

2. Ministério da Saúde. Brasil. Informações em Saúde - Mortalidade. [online] [citado 2000 abr 4] Disponível em: http://www.saude.gov.br/inform/ indica/indica

3. III Diretrizes brasileiras sobre dislipidemias e diretriz de prevenção de aterosclerose do Departamento de Aterosclerose da Sociedade Brasileira de Cardiologia. Arq Bras Cardiol 2001; 77 Supl 3:S1-S48.

4. Grundy SM, et al. Primary prevention of coronary heart disease: guidance from Framingham: a statement for healthcare professionals from the AHA task force on risk reduction. Circulation 1998; 97:1876-87.

5. Armaganijan D, Batlouni M. Impacto dos fatores de risco tradicionais. Rev Soc Cardiol 2000; 10(6):686-93.

6. Mendonça GAS. Tendência de investigação epidemiológica em doenças crônicas. Cad Saude Publica 2001; 17(3):697-703.

7. Krauss RM, et al. AHA Dietary guidelines. Revision 2000: a statement for healthcare professionals from the Nutrition Committee of the American Heart Association. Circulation 2000; 102(31):2284-99.

8. National Cholesterol Education Program. Summary of the Third Report of the National Cholesterol Education Program Expert Panel on Detection, Evaluation, and Treatment of High Blood 
Cholesterol in Adults (Adults Treatment Panel III). JAMA 2001; 285:2486-97.

9. World Health Organization. Physical Status: the use and interpretation of anthropometry. Geneva: WHO; 1995. Report of a WHO Expert Commitee. WHO Report Series 854.

10. World Health Organization. Obesity: preventing and managing the global epidemic. Geneva: WHO; 1998. Report of a WHO Consultation on Obesity.

11. Janssen I, Heymsfield SB, Allison DB, Kotler DP, Ross R. Body mass index and waist circumference independently contribute to the prediction of nonabdominal, abdominal subcutaneous, and visceral fat. Am J Clin Nutr 2002; 75:683-8.

12. Bray GA. Classification and evaluation of the obesities. Med Clin North Am 1989; 73:161-84.

13. Lee JS, Aoki K, Kawakubo K, Gunji A. A study on indices of body fat distribution for screening for obesity. Sangyo Eiseigkaku Zasshi 1995; 37:9-18.

14. Lean MEJ, Han TS, Morrison CE. Waist circumference as a measure for indicating need for weight management. Br Med J 1995; 311:158-61.

15. Egger $G$. The case for using waist to hip ratio measurements in the routine medical checks. Med J Aust 1992; 156:280-5.

16. Beegon R, Beegon R, Niaz MA, Singh RB. Diet, central obesity and prevalence of hypertension in the urban population of South India. Int J Cardiol 1995; 51:183-91.

17. Marti B, Tuomilehto J, Salomaa V, Karto-Vaara L, Korhonen $\mathrm{H}$ J, Pietinen $\mathrm{P}$. Body fat distribution in the Finnish population: environmental determinants and predictive power for cardiovascular risk factor levels. J Epidemiol Comm Health 1991; 45:131-7.

18. Keenan NI, Strogatz DS, James SA, Ammerman $A S$, Rice BL. Distribution and correlates of waist-to-hip ratio in black adults: the Pitt County Study. Am J Epidemiol 1992; 135(6):678-84.

19. Pereira R, Sichieri R, Marins VMR. Razão cintura/ quadril como preditor de hipertensão arterial. Cad Saude Publica 1999; 15(2):333-44.
20. Lerario DDG, Gimeno SG, Franco LJ, lunes M, Ferreira SRG. Excesso de peso e gordura abdominal para síndrome plurimetabólica em nipo-brasileiros. Rev Saude Publica 2002; 36(1):4-11.

21. Conway JM, Chatnetsa FF, Wang P. Intraabdominal adipose tissue and anthropometric surrogates in African American women with upper and lower body obesity. Am J Clin Nutr 1997; 66:1345-51.

22. Han TS, Van Leer EM, Seidll JC, Lean ME. Waist circumference action levels in the identification of cardiovascular risk factors: prevalence study in a random sample. Br Med J 1995; 311:1401-5.

23. Velásquez-Meléndez G, Kac G, Valente JG, Tavares R, Silva CQ, Garcia ES. Evaluation of waist circumference to predict general obesity and arterial hypertension in women in Greater Metropolitan Belo Horizonte, Brazil. Cad Saude Publica 2002; 18(3):765-71.

24. Cervato AM, Mazzilli RN, Martins IS, Marucci MFN. Dieta habitual e fatores de risco para doenças cardiovasculares. Rev Saude Publica 1997; 31(3):227-35.

25. Mustad VA, Kris-Etherton PM. Além da redução do colesterol: decifrando os benefícios da intervenção alimentar para a doença cardiovascular. Curr Atheroscler Reports Brasil 2001; 1:2-7.

26. Schaefer EJ. Lipoproteins, nutrition, and heart disease. Am J Clin Nutr 2002; 75(2): 191-212.

27. Martins IS, et al. Hábitos alimentares aterogênicos de grupos populacionais em área metropolitana da região sudeste do Brasil. Rev Saude Publica 1994; 28(5):349-56.

28. Parada NM, Cozza E, Parada JL. Relación entre hábitos alimentarios y niveles de colesterol serico en una populación suburbana de Argentina. Arch Latinoam Nutr 1999; 49(4):333-7.

29. Fornes NS, et al. Food frequency consumption and Lipoproteins serum levels in the population of na urban area, Brazil. Rev Saude Publica 2000; 34(4):380-7.

30. Guedes DP, Guedes JERP. Physical activity, cardiorespiratory fitness, dietary content, and risk factor that cause a predisposition towards 
cardiovascular disease. Arq Bras Cardiol 2001; 77(3):251-7.

31. Delgado M, Gutierrez A, Cano MD. Elimination of meat, fish and derived products from the Spanish-Mediterranean diet: effect on the plasma lipid profile. Ann Nutr Metab 1996; 40:202-11.

32. Rajaram S, et al. A Monounsaturated Fatty Acid: rich pecan enriched diet favorable alters the serum lipid profile of healthy men and women. J Nutr 2001; 131:2275-9.

33. Dewailly $E$, et al. Relations between n-3 Fatty-Acid status and cardiovascular disease risk factors among Quebecers. Am J Clin Nutr 2001; 74(5):603-11.

34. Djossé $\mathrm{L}$, et al. Relations between dietary Linolenic Acid and coronary artery disease in the National Heart, Lung, and Blood Institute Family Heart Study. Am J Clin Nutr 2001; 74(5):612-9.

35. Oliveira SP, Tahin QS, Cavalcanti TC. Epidemiologia das doenças isquêmicas do coração: papel da dieta. Rev Nutr PUCCAMP 1991; 4(1/2):146-53.

36. Fornes NS, Martins IS, Velásquez-Meléndez G, Latorre MRDO. Escores de consumo alimentar e níveis lipêmicos em população de São Paulo, Brasil. Rev Saude Publica 2002; 36(1):12-8.

37. Keys $A$. The diet and 15-year death rate in the seven countries study. Am J Epidemiol 1986; 124:903-15.

38. Monteiro JBR, Rosado LEFL. Nutrição e doenças cardiovasculares. Viçosa: Imprensa Universitária; 1993.

39. Santos TM. Lipídios. In: Dutra-de-Oliveira JE, Marchini JS. Ciências nutricionais. São Paulo: Sarvier; 1998. p.87-97.

40. Mustad VA, et al. Reducing saturated fat intake is associated with increased levels of LDL receptors on mononuclear cells in healthy men and women. J Lipid Res 1997; 38:459-68.
41. Caggiula AW, Mustad VA. Effects of dietary fat and fatty acids on coronary artery disease risk and total and lipoprotein cholesterol concentrations: epidemiologic studies. Am J Clin Nutr 1997; 65(Suppl):1597s-610s.

42. Mattson FH, Erickson BA, Kligman AM. Effect of dietary cholesterol on serun cholesterol in man. Am J Clin Nutr 1972; 25:589-94.

43. Moura EC, Sonati JG. Perfil lipídico de dietas e sua relação com os níveis de colesterolemia em escolares de uma escola pública de Campinas, São Paulo [Brasil]. Rev Nutr 1998; 11(1):69-75.

44. Schulte H, Cullen P, Assmann G. Obesity, mortality and cardiovascular disease in the Munster Heart Study (PROCAN). Atherosclerosis 1999; 144:199-209.

45. Kannel WB. The Framingham Study: its 50 years legacy and future promise. J Atheroscler Thromb 2000; 6:60-6.

46. Bertolami MC. A conexão entre as lipoproteínas e a aterosclerose. Rev Soc Cardiol 2000; 10(6):694-9.

47. Gold AL, et al. Cholesterol reduction yields clinical benefit: impact of statin trials. Circulation 1998; 97:946-52.

48. Santos RD, Maranhão RC. Importância da lipoproteína(a) na aterosclerose. Rev Soc Cardiol 2000; 10(6):723-7.

49. Danesh J, et al. Low grade inflammation and coronary heart disease: prospective study and updated meta-analyses. Br Med J 2000; 321:199-204.

50. Ridker PM. High-Sensitivity C-Reactive protein: potential adjunct for global risk assessment in the primary prevention of cardiovascular disease. Circulation 2001; 103:1813-8.

Recebido para publicação em 4 de dezembro de 2002 e aceito em 30 de outubro de 2003. 